\title{
An Approach to the Generation of SPICE Models Feasible for EMC Problems
}

\author{
Jan Carlsson \\ SP Swedish National Testing \& Research Institute \\ Brinellgatan 4 \\ SE-501 15, Borås, SWEDEN
}

\author{
Urban Lundgren \\ Luleå University of Technology \\ Regnbågsallén \\ SE-971 87, Luleå, SWEDEN
}

\begin{abstract}
A method to describe barriers such as filters, cables, connectors etc. with circuits consisting of linear discrete components is presented. The circuit is constructed by viewing the barrier as a multi-conductor transmission line for which the per-unit length parameters have to be determined. A developed two-dimensional finite difference program in which the cross-section is defined by drawing it using the CAD-like user interface computes these. For barriers that cannot be viewed as transmission lines a method for determining equivalent circuits outgoing from measured S-parameters has been developed. Derived models have been used in SPICE and validated by comparison with measurements.
\end{abstract}

\section{Introduction}

When analyzing EMC problems for complex systems it is necessary to break down the system and characterize each coupling path or barrier. By describing a barrier with a circuit consisting of discrete components the propagation of disturbances in the system can be computed by the use of an ordinary circuit simulator as e.g. SPICE [1]. However, the main problem is to find a circuit representation and component values that describe the behavior of the barrier. Two different approaches to accomplish this have been used.

For barriers that can be viewed as multi-conductor transmission lines the cross-section, which is assumed to be uniform, is divided into small elements and the Laplace equation is solved. From the solution the charges on each conductor can be computed and thereby the per-unit length inductance and capacitance matrices. When the matrices are known it is a straightforward task to construct a circuit representation that can be used in a standard circuit simulator.

Barriers such as e.g. commercial filters that cannot be viewed as transmission lines must be treated in another way. In the method that we have used the first step is to set up a discrete circuit and then compute the S-parameters. The computed Sparameters are then compared with measured for the frequency range of interest and the weighted difference is minimized by adjusting component values. This is done in an iterative scheme searching among component values in a given range.

\section{Determination of the per-unit length parameters}

For some type of barriers it is sufficient to have knowledge of the geometrical shape and the material in the cross-section in order to describe its electrical characteristics. One example would e.g. be the barrier between two parallel conductors on a printed circuit board for which the crosstalk could be computed with the knowledge of the geometry and the material properties of the circuit board. The approach that we have used for these types of barriers is to first determine the per-unit length parameters and then create a discrete circuit representation. The circuit can then be used in a standard circuit simulator such as e.g. SPICE to obtain the desired responses either in the time or in the frequency domain. The requirement for this method to be successful is that the barrier under consideration can be viewed as a multi-conductor transmission line, i.e. it must have a uniform cross-section with an extent that is small compared to the wavelength. For barriers that don't have a uniform cross-section it is sometimes possible to describe them as a number of cascaded sections with uniform cross-sections, i.e. a staircase approximation.

Since the approach that we have used is based on multiconductor transmission line theory we have to determine the per-unit length parameters in order to arrive at the wanted circuit representation. Looking at the circuit representation of a short section of a multi-conductor transmission with three wires (reference not counted), Figure 1, the following relations between the entries in the per-unit length capacitance and inductance matrices and the values of the circuit elements can be found.

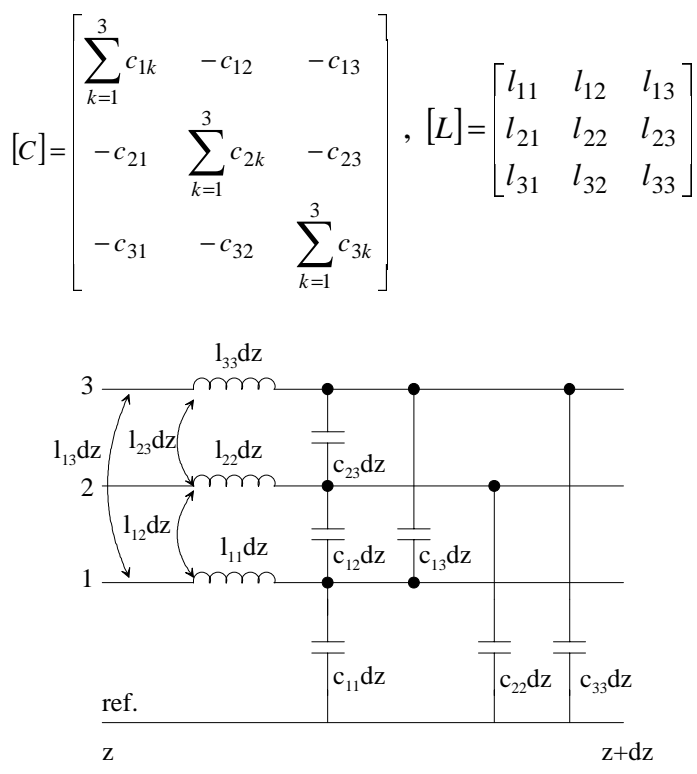

Figure 1 Circuit representation of a short section of a three-wire transmission line. 
The per-unit length parameters can be computed by using numerical methods such as the method of moments (MoM) [2], the finite element method (FEM) [3] etc. For some simple cases it is even possible to use analytical formulas. The method that we have used is the finite difference method (FDM) [4, Sec. 3], mainly because it is simple to implement and that it easily can handle complicated cross-sections with different materials. For the simple example shown in Figure 2 the dimension of the per-unit length capacitance and inductance matrices will be two by two, since we have two conductors and a common reference (the ground plane). For the general case the $i j$-element in the capacitance matrix can be determined by letting the potential on all conductors except the $\mathrm{j}$ :th be equal to zero and evaluating the charge on the $\mathrm{i}:$ th conductor, i.e. [5], $C_{i j}=\left.\frac{Q_{i}}{V_{j}}\right|_{V_{m}=0, m \neq j}$. Thus, in order to determine the capacitance matrix for the PCB in Figure 2, we have to solve the Laplace equation for the configuration two times with different boundary conditions.

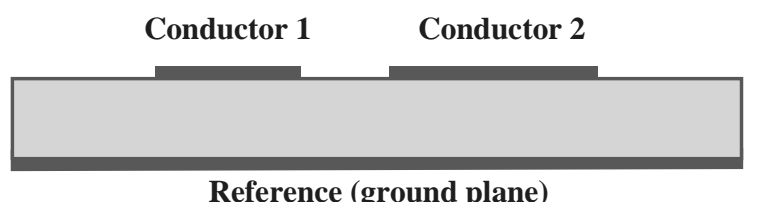

Figure 2 Cross-section of a printed circuit board with two conductors.

The solution of the Laplace equation gives the potential distribution in the region and we can by applying Gauss' law determine the charge per unit-length on conductor $i$ as: $Q_{i}=-\int_{l_{i}} \varepsilon \nabla V \cdot \hat{n} d l_{i}$ where $l_{i}$ is a closed line around conductor $i$, $n$ is an outward directed unit vector and $V$ is the potential distribution. The inductance matrix can be computed by the knowledge of the capacitance matrix for the case when all material in the cross section is free space, i.e. [6], $[L]=\mu_{0} \varepsilon_{0}\left[C_{0}\right]^{-1}$ where $\left[C_{0}\right]$ is the capacitance matrix when all dielectric material in the cross section is replaced by free space. The remaining problem now is to determine the potential distribution by solving the Laplace equation. By starting with Maxwell's equations for the two-dimensional electrostatic case and approximating the derivatives with finite differences we can quite easily write down the following relation between the potential in neighboring nodes in the finite difference mesh, see Figure 3.

$$
\begin{aligned}
& V_{i, j}=\frac{V_{i+1, j}\left(\varepsilon_{A}+\varepsilon_{B}\right)}{2\left(\varepsilon_{A}+\varepsilon_{B}+\varepsilon_{C}+\varepsilon_{D}\right)}+\frac{V_{i-1, j}\left(\varepsilon_{C}+\varepsilon_{D}\right)}{2\left(\varepsilon_{A}+\varepsilon_{B}+\varepsilon_{C}+\varepsilon_{D}\right)} \\
& +\frac{V_{i, j+1}\left(\varepsilon_{B}+\varepsilon_{C}\right)}{2\left(\varepsilon_{A}+\varepsilon_{B}+\varepsilon_{C}+\varepsilon_{D}\right)}+\frac{V_{i, j-1}\left(\varepsilon_{A}+\varepsilon_{D}\right)}{2\left(\varepsilon_{A}+\varepsilon_{B}+\varepsilon_{C}+\varepsilon_{D}\right)}
\end{aligned}
$$

By giving all nodes in the cross-section an initial estimate, $V_{i, j}^{0}$, and by scanning through the nodes by an iterative procedure we can determine the potential distribution in the whole region and thereby are we able to compute the per-unit length parameters.

\section{Computer code for determining per-unit length parameters}

Based on the method for computing the per-unit length parameters described above a computer code called FD2D was developed. The code has a Windows user interface where the cross-section of the barrier easily can be defined by simply drawing it on the screen, see Figure 5. The code has been validated against several test cases and the agreement has been found to be good with previously published results. As an example of a simple validation the characteristic impedance for an air coaxial cable with an inner to outer conductor radius ratio of five was computed using a grid size of 200 by 200 nodes. The computed impedance was $96.85 \Omega$, which should be compared to the exact value of $96.57 \Omega$.

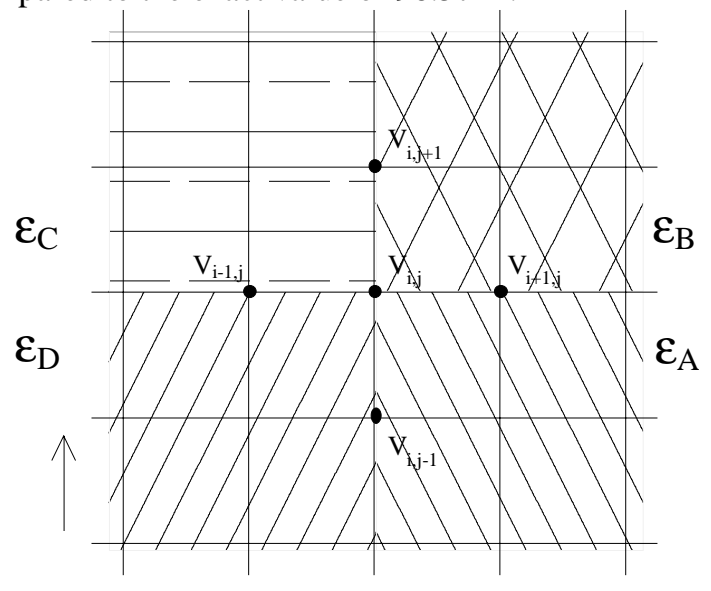

Figure 3 A part of the finite difference mesh.

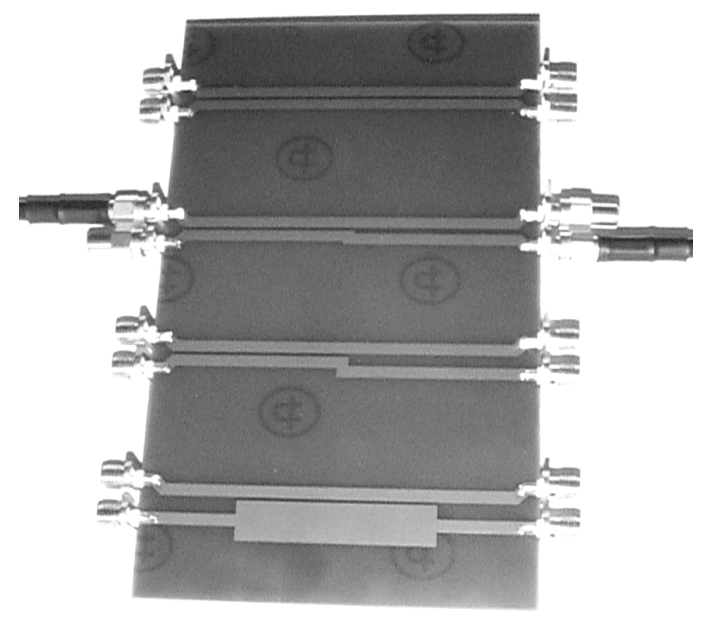

Figure 4 Printed circuit board for measurements of crosstalk between adjacent conductors. 


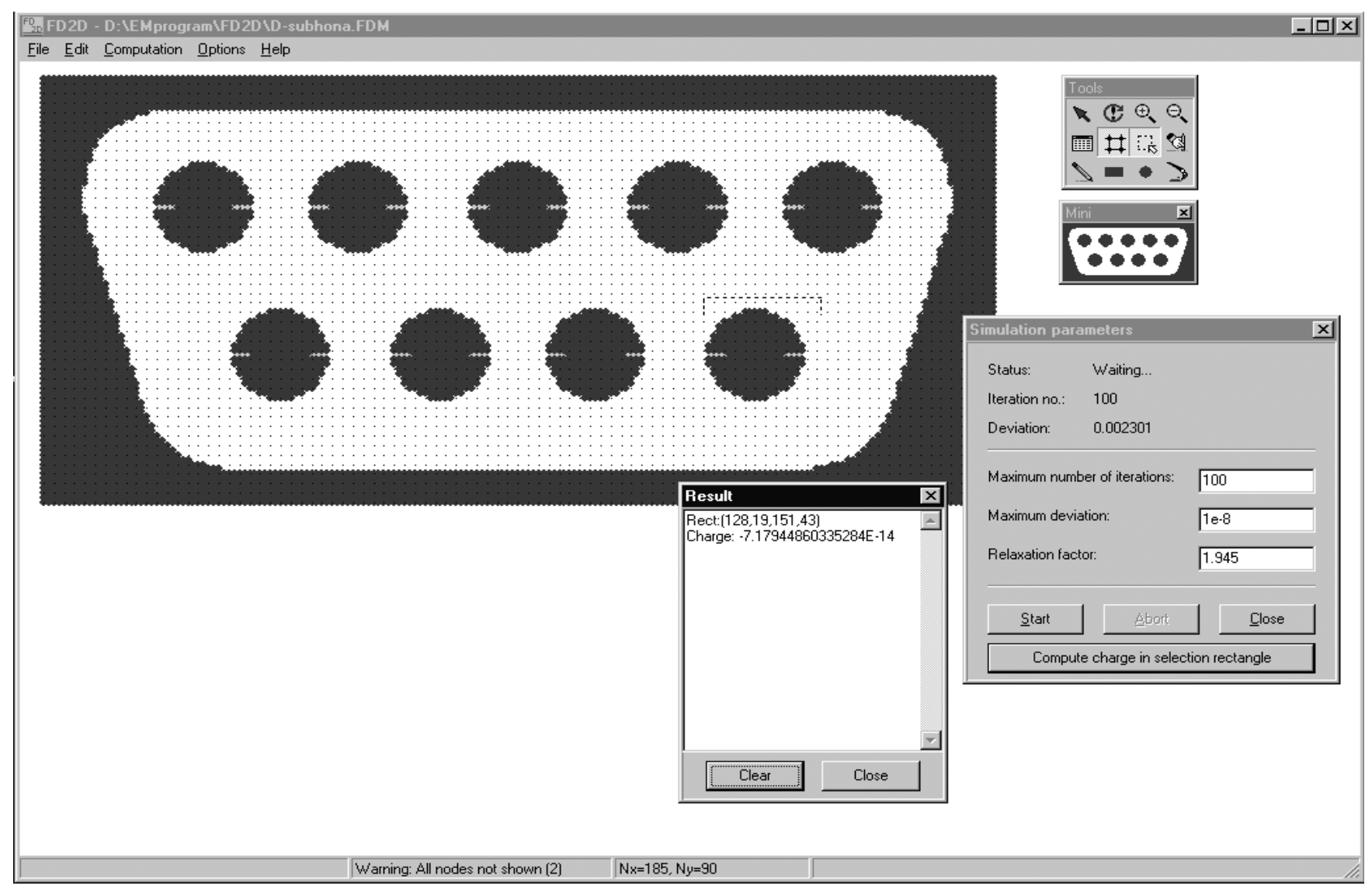

Figure 5 User interface of the finite difference program for determination of per-unit length parameters. In the example a D-sub connector is analysed.

By defining the length of the analyzed barrier the code is capable of generating a representative SPICE circuit file or computing the scattering parameters (S-parameters) for a given frequency range. The $\mathrm{S}$-parameters are determined by first setting up the chain matrix for the analyzed device [7] and then using relations between the chain matrix and the scattering matrix. These relations can easily be found by expressing the total voltages and currents in terms of the scattering voltages and currents, see (2).

\section{Validation against measurements}

In order to validate the computed response of a barrier by using the FD2D program for generating circuit files and then using them in SPICE a number of measurements have been conducted. As an example the crosstalk between conductors on a printed circuit board was analyzed. For the measurement of near end and far end crosstalk a vector network analyzer was used to get the scattering parameters from the four-port made of two adjacent conductors with the length $100 \mathrm{~mm}$ across the circuit board. All four ports were connected to $50 \Omega$ during all measurements. Configurations with different spacing between the conductors were measured and compared with simulations on the corresponding circuit models. Also conductors with a non-uniform cross section along the length were considered, see Figure 4.

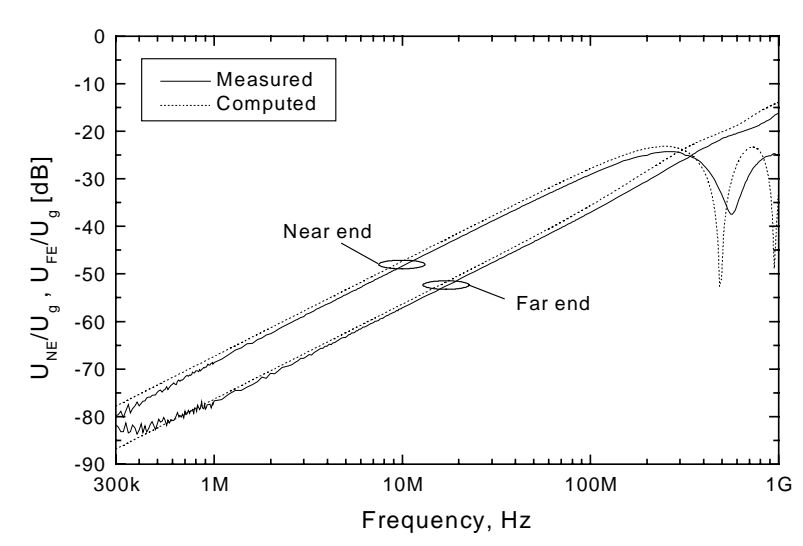

Figure 6 Crosstalk between conductors on a printed circuit board determined by modeling the barrier as a discrete circuit compared to measurements.

One example of the agreement between the computed crosstalk and the measured crosstalk is shown in Figure 6. As can be seen in the figure the agreement is good up to $1 \mathrm{GHz}$, although the resonance frequencies are not accurately predicted. One reason for this is that in the circuit model approach that we have used radiation losses are not taken into account. 


\section{Barriers that cannot be viewed as transmission lines}

For some types of barriers measurement is the only possible way of characterizing the behavior. For these cases a method by which it is possible to deduce a network from measured data has been developed. In this approach we take the measured S-parameters and compute the same for an assumed network. The next step is to seek for optimal values for components in the network so that a best fit, in some respect, is found. By this procedure the wanted network representing the measured device can be determined. Of course, the success of this method is only guaranteed if the assumed network actually can represent the device under consideration. Thus, a basic knowledge of circuit theory and some experience are required.

Since measurements with a network analyzer usually gives the S-parameters of a network it is natural to use these parameters as the basis for the comparison with the assumed network. Thus, we have to compute the S-parameters for the network. This can be done by first computing the chain matrix for the network and then convert to S-parameters. The relations between the $\mathrm{S}$-parameters and the chain parameters are:

$$
\begin{aligned}
& {\left[S_{11}\right]=\left[[1]+\left([A]+\frac{1}{Z_{0}}[B]\right)\left(Z_{0}[C]+[D]\right)^{-1}\right]^{-1} *} \\
& {\left[\left([A]+\frac{1}{Z_{0}}[B]\right)\left(Z_{0}[C]+[D]\right)^{-1}-[1]\right]} \\
& {\left[S_{12}\right]=\left[[1]+\left([A]+\frac{1}{Z_{0}}[B]\right)\left(Z_{0}[C]+[D]\right)^{-1}\right]^{-1} *} \\
& {\left[\left([A]-\frac{1}{Z_{0}}[B]\right)-\left([A]+\frac{1}{Z_{0}}\right)\left(Z_{0}[C]+[D]\right)^{-1}\left(Z_{0}[C]-[D]\right)\right]} \\
& {\left[S_{21}\right]=2\left[[1]+\left(Z_{0}[C]+[D]\right)^{-1}\left([A]+\frac{1}{Z_{0}}[B]\right)\right]^{-1}\left(Z_{0}[C]+[D]\right)^{-1}} \\
& {\left[S_{22}\right]=-\left[[1]+\left(Z_{0}[C]+[D]\right)^{-1}\left([A]+\frac{1}{Z_{0}}[B]\right)\right]^{-1}\left(Z_{0}[C]+[D]\right)^{-1} *} \\
& {\left[\left([A]-\frac{1}{Z_{0}}[B]\right)+\left(Z_{0}[C]-[D]\right)\right]}
\end{aligned}
$$

where $[A],[B],[C]$ and $[D]$ are the usual sub-matrices in the general chain matrix.

When the S-parameters have been determined for all frequencies of interest we have to compare the values with measured S-parameters. Figure 7 shows an example of such a comparison.

In order to find component values in the network that will make the network representing the measured device we minimize the average difference between measured and computed S-parameters, i.e. we minimize the function $Q=\sum_{i}\left|\Delta_{i}(f)\right|$.

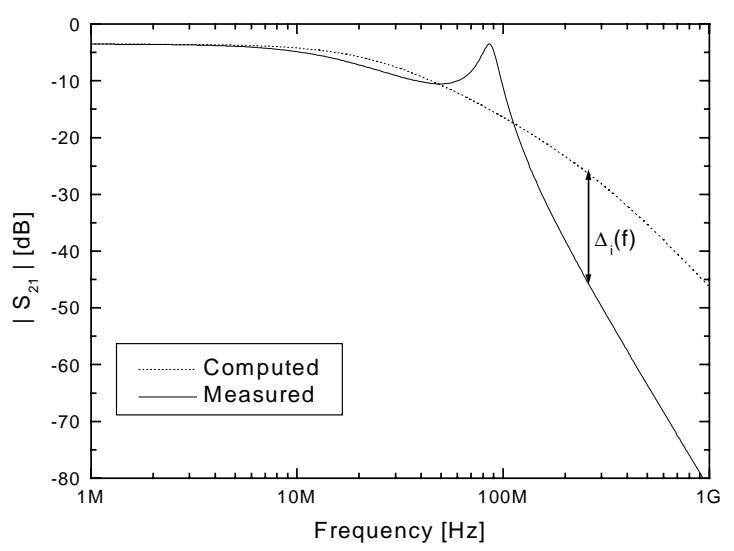

Figure 7 Example of computed and measured Sparameter.

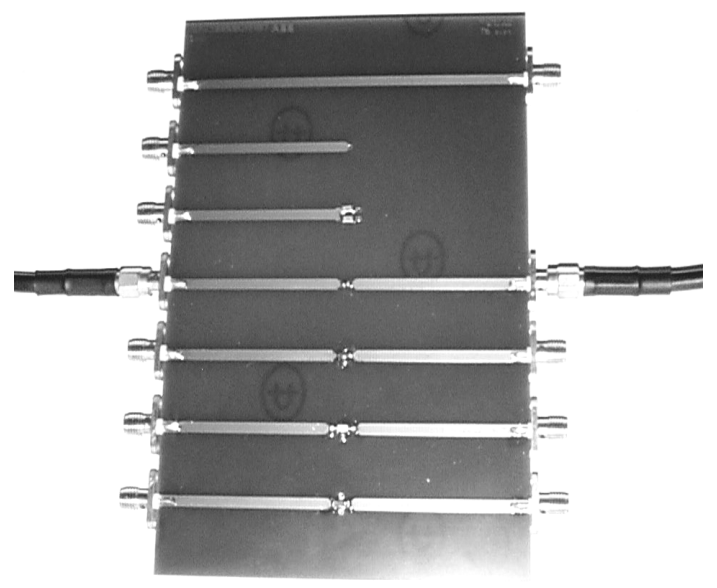

Figure 8 Printed circuit board for the characterization of commercial surface mounted filters with three calibration traces and four different filters.

In order to validate the method a number of different surface mounted filters were studied. The filters were placed on a special circuit board, Figure 8, on which also conductors for calibrating the network analyzer were present. The measurement procedure that was used involves calibration to the footprint of the examined component by a through, reflection and match calibration method. When the calibration was done the four $S$-parameters $S_{11}, S_{21}, S_{12}$ and $S_{22}$ for each surface mounted filter were measured. 


\section{Lumped circuit generation for a feed through capacitor filter}

For one of the studied commercial filters of the type feed through capacitor the error function minimization procedure explained above gave component values to the assumed network resulting in a lumped circuit model shown in Figure 9. The schematic also shows a $50 \Omega$ generator and a matched load used when running the SPICE circuit simulation.

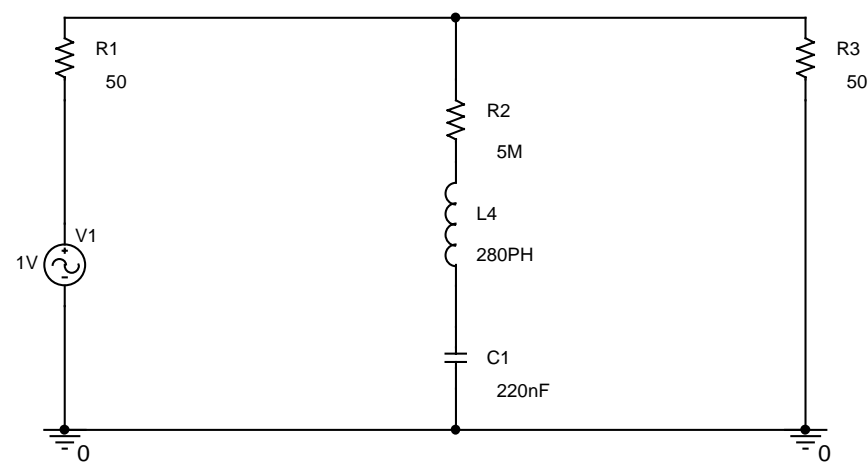

Figure 9 A derived lumped circuit model of a feed through filter used in the computation (note that in SPICE syntax $M$ or $\mathbf{m}$ stands for the prefix milli-).

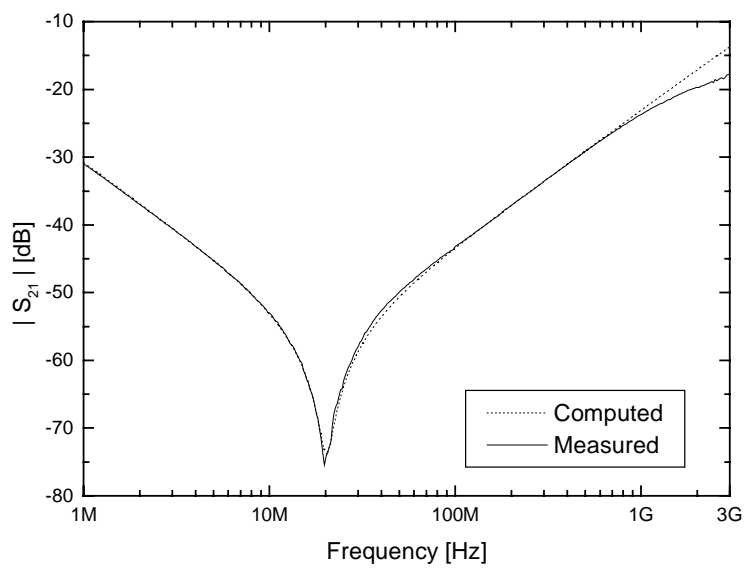

Figure 10 Insertion loss for a feed through filter determined using a circuit model and measurements.

With this developed lumped circuit description of the feed through capacitor filter the behavior of the filter can easily be simulated in an electronic circuit simulator such as SPICE. In Figure 10 the results from a simulation of insertion loss is shown. For comparison the measured insertion loss for the commercial filter is shown in the same graph.

\section{Lumped circuit generation for a series inductance filter}

A surface mounted series inductor filter was included in the study. The assumed network for this filter was enhanced stepwise by adding discrete components one at a time. The lumped circuit model for the filter finally chosen is shown in Figure 11 together with the generator and load used in simulation.

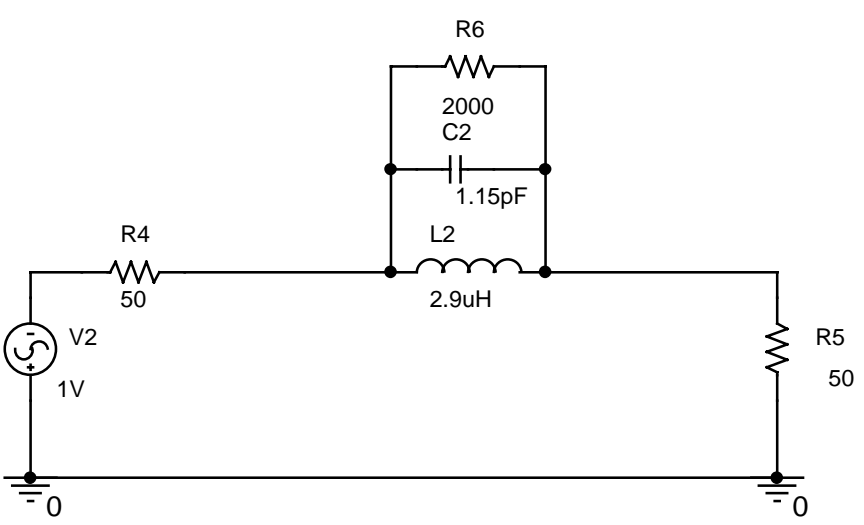

Figure 11 A derived lumped circuit model of a series inductance filter used in the computation.

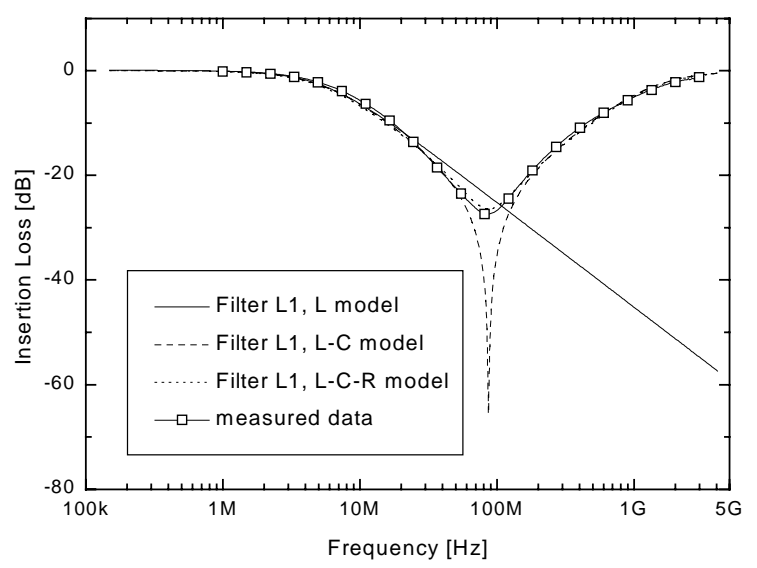

Figure 12 Insertion loss for a series inductance filter determined using different circuit models and measurements.

The different lumped circuit models with increasing complexity were used when simulating the insertion loss of the filter in a circuit simulator. The resulting simulated insertion loss is shown in Figure 12 together with measured values. As can been seen in Figure 12 the first two attempts to a network design both failed to agree with measured data at a frequency close to $100 \mathrm{MHz}$. The third attempt to choose a network capable of adapting to the correct behavior was more successful. After minimizing the error function for this network a reasonable good fit to the measured insertion loss was obtained. In this case there is a very good agreement between the developed lumped circuit model of the filter and the measurements up to at least $4 \mathrm{GHz}$. 


\section{Conclusions}

The developed methods for generating lumped circuit models for transmission like barriers as well as for barriers with unknown geometrical shape have been verified against measurements on prototype printed circuit boards. Good agreement between SPICE simulation and measurement was found for frequencies up to $1 \mathrm{GHz}$. For models of transmission like barriers the upper frequency limit for good agreement is depending on the number of cascaded sections in the circuit model. For the $100 \mathrm{~mm}$ parallel conductor crosstalk, an agreement within a few $\mathrm{dB}: \mathrm{s}$ up to $1 \mathrm{GHz}$ was achieved with 16 cascaded sections. The method of adapting an assumed network to measured S-parameters gave an good agreement up to 1 to $4 \mathrm{GHz}$ when simple networks were assumed. Better agreement in the high frequency range can of course be reached by assuming a more complex network.

\section{Acknowledgements}

Thanks to Lennart Hasselgren at IVF (The Swedish Institute of Production Engineering Research) for running SPICE simulations with the generated lumped circuit models.

\section{References}

[1] L. W. Nagel, "SPICE2: A Computer Program to Simulate Semiconductor Circuits", Memorandum No. M520, May 1975.

[2] R.F. Harrington, "Field computation by moment methods", Macmillan New York 1968.

[3] P.P. Silvester and R.L. Ferrari, "Finite elements for electrical engineers", 2nd ed., Cambridge University Press, 1990.

[4] Pei-bai Zhou, "Numerical Analysis of Electromagnetic Fields", Springer-Verlag 1993.

[5] R. Laroussi and G. I. Costache, "Finite-Element Method Applied to EMC Problems", IEEE Transactions on Electromagnetic Compatibility, Vol. 35, No. 2, pp. 178-184, May 1993.

[6] C. Wei, R. F. Harrington, J. R. Mautz and T. K. Sarkar., "Multiconductor Transmission Lines in Multilayered Dielectric Media", IEEE Transactions on Microwave Theory and Techniques, Vol. 32, No. 4, pp. 439-450, April 1984.

[7] F. M. Tesche, M. V. Ianoz and T. Karlsson, "EMC analysis methods and computational models", John Wiley \& Sons, inc., 1997. 